\title{
Light-by-Light Scattering Constraint on Born-Infeld Theory
}

\author{
John Ellis \\ Theoretical Particle Physics and Cosmology Group, Physics Department, \\ King's College London, London WC2R 2LS, United Kingdom \\ and Theoretical Physics Department, CERN, CH-1211 Geneva 23, Switzerland \\ Nick E. Mavromatos \\ Theoretical Particle Physics and Cosmology Group, Physics Department, \\ King's College London, London WC2R 2LS, United Kingdom
}

Tevong You

DAMTP, University of Cambridge, Wilberforce Road, Cambridge CB3 OWA, United Kingdom and Cavendish Laboratory, University of Cambridge, J.J. Thomson Avenue, Cambridge CB3 OHE, United Kingdom

(Received 28 March 2017; revised manuscript received 2 May 2017; published 27 June 2017)

\begin{abstract}
The recent measurement by ATLAS of light-by-light scattering in $\mathrm{LHC} \mathrm{Pb}-\mathrm{Pb}$ collisions is the first direct evidence for this basic process. We find that it excludes a range of the mass scale of a nonlinear Born-Infeld extension of QED that is $\lesssim 100 \mathrm{GeV}$, a much stronger constraint than those derived previously. In the case of a Born-Infeld extension of the standard model in which the $\mathrm{U}(1)_{Y}$ hypercharge gauge symmetry is realized nonlinearly, the limit on the corresponding mass reach is $\sim 90 \mathrm{GeV}$, which, in turn, imposes a lower limit of $\gtrsim 11 \mathrm{TeV}$ on the magnetic monopole mass in such a $\mathrm{U}(1)_{Y}$ Born-Infeld theory.
\end{abstract}

DOI: 10.1103/PhysRevLett.118.261802

Over 80 yr ago, soon after Dirac proposed his relativistic theory of the electron [1] and his interpretation of "hole" states as positrons [2], Halpern [3] in 1933 and Heisenberg [4] in 1934 realized that quantum effects would induce light-by-light scattering, which was first calculated in the low-frequency limit by Euler and Kockel [5] in 1935. Subsequently, Heisenberg and Euler [6] derived in 1936 a more general expression for the quantum nonlinearities in the Lagrangian of quantum electrodynamics (QED), and a complete calculation of light-by-light scattering in QED was published by Karplus and Neuman [7] in 1951. However, measurement of light-by-light scattering has remained elusive until very recently. In 2013 d'Enterria and da Silveira [8] proposed looking for light-by-light scattering in ultraperipheral heavy-ion collisions at the LHC, and evidence for this process was recently presented by the ATLAS Collaboration [9], at a level consistent with the QED predictions in Refs. $[8,10]$.

In parallel with the early work on light-by-light scattering in QED, and motivated by a "unitarian" idea that there should be an upper limit on the strength of the electromagnetic field, Born and Infeld [11] proposed in 1934 a

Published by the American Physical Society under the terms of the Creative Commons Attribution 4.0 International license. Further distribution of this work must maintain attribution to the author(s) and the published article's title, journal citation, and DOI. conceptually distinct nonlinear modification of the Lagrangian of QED:

$$
\begin{aligned}
\mathcal{L}_{\mathrm{QED}} & =-\frac{1}{4} F_{\mu \nu} F^{\mu \nu} \rightarrow \\
\mathcal{L}_{\mathrm{BI}} & =\beta^{2}\left(1-\sqrt{1+\frac{1}{2 \beta^{2}} F_{\mu \nu} F^{\mu \nu}-\frac{1}{16 \beta^{4}}\left(F_{\mu \nu} \tilde{F}^{\mu \nu}\right)^{2}}\right),
\end{aligned}
$$

where $\beta$ is an a priori unknown parameter with the dimension of $[\mathrm{mass}]^{2}$ that we write as $\beta \equiv M^{2}$, and $\tilde{F}_{\mu \nu}$ is the dual of the field strength tensor $F_{\mu \nu}$. Interest in BornInfeld theory was revived in 1985 when Fradkin and Tseytlin [12] discovered that it appears when an Abelian vector field in four dimensions is coupled to an open string, as occurs in models inspired by $M$ theory in which particles are localized on lower-dimensional "branes" separated by a distance $\simeq 1 / \sqrt{\beta}=1 / M$ in some extra dimension. (Remarkably, the maximum field strength is related to the fact that the brane velocity is limited by the velocity of light [13], confirming the insight of Born and Infeld [11].) Depending on the specific brane scenario considered, $M$ might have any value between a few hundred $\mathrm{GeV}$ and the Planck scale $\sim 10^{19} \mathrm{GeV}$. For the purposes of this Letter, we consider only the relevant terms of fourth order in the gauge field strengths in Eq. (1).

To date, there has been no strong lower limit on the Born-Infeld scale $\beta$ or, equivalently, the brane mass scale $M$ and the brane separation $1 / M$. A constraint corresponding 
to $M \gtrsim 100 \mathrm{MeV}$ was derived in Ref. [14] from electronic and muonic atom spectra, though the derivation was questioned in Ref. [15]. Measurements of photon splitting in atomic fields [16] were considered in Ref. [17], where it was concluded that they provided no limit on the BornInfeld scale and it was suggested that measurements of the surface magnetic field of neutron stars [18] might be sensitive to $M=\sqrt{\beta} \sim 1.4 \times 10^{-5} \mathrm{GeV}$. More recently, measurements of nonlinearities in light by the PVLAS Collaboration [19] were somewhat more sensitive to the individual nonlinear terms in Eq. (1), but they were not sensitive to the particular combination appearing in the Born-Infeld theory, as discussed in Ref. [20], where more references can be found.

Here, we show that the agreement of the recent ATLAS measurement of light-by-light scattering with the standard QED prediction provides the first limit on $M$ in the multi$\mathrm{GeV}$ range, excluding a significant range extending to

$$
M \gtrsim 100 \mathrm{GeV},
$$

entering the range of interest to brane theories. This limit is obtained under quite conservative assumptions, and plausible stronger assumptions would strengthen our lower bound to $M \gtrsim 200 \mathrm{GeV}$.

One may also consider a Born-Infeld extension of the standard model in which the hypercharge $\mathrm{U}(1)_{Y}$ gauge symmetry is realized nonlinearly, in which case the limit (2) is relaxed to

$$
M_{Y}=\cos \theta_{W} M \gtrsim 90 \mathrm{GeV},
$$

where we have used $B_{Y}^{\mu}=\cos \theta_{W} A_{\mathrm{EM}}^{\mu}-\sin \theta_{W} Z^{\mu}$ and $\sin ^{2} \theta_{W} \simeq 0.23$, with $\theta_{W}$ being the weak mixing angle. As a corollary of this lower limit on the $\mathrm{U}(1)_{Y}$ brane scale, we recall that Arunasalam and Kobakhidze recently pointed out [21] that the standard model modified by a Born-Infeld $\mathrm{U}(1)_{Y}$ theory has a finite-energy electroweak monopole $[22,23]$ solution $\mathcal{M}$, whose mass they estimated as $M_{\mathcal{M}} \simeq 4 \mathrm{TeV}+72.8 M_{Y}$. Such a monopole is less constrained by Higgs measurements than electroweak monopoles in other extensions of the standard model [24], and hence of interest for potential detection by the ATLAS [25], CMS, and MoEDAL experiments at the LHC [26]. However, our lower limit $M_{Y} \gtrsim 90 \mathrm{GeV}$ (2) corresponds to a $95 \%$ C.L. lower limit on the mass of this monopole $M_{\mathcal{M}} \gtrsim 11 \mathrm{TeV}$, excluding its production at the LHC.

Following the suggestion of Ref. [8], we consider ultraperipheral heavy-ion collisions in which the nuclei scatter quasielastically via photon exchange: $\mathrm{Pb}+\mathrm{Pb}$ $(\gamma \gamma) \rightarrow \mathrm{Pb}^{(*)}+\mathrm{Pb}^{(*)}+X$, as depicted in Fig. 1, effectively acting via the equivalent photon approximation (EPA) [27] as a photon-photon collider. (The authors of Ref. [8] also considered the observability of light-by-light scattering in $p p$ collisions at the LHC but concluded that they were less promising than $\mathrm{Pb}-\mathrm{Pb}$ collisions.) The EPA allows the electromagnetic field surrounding a highly relativistic

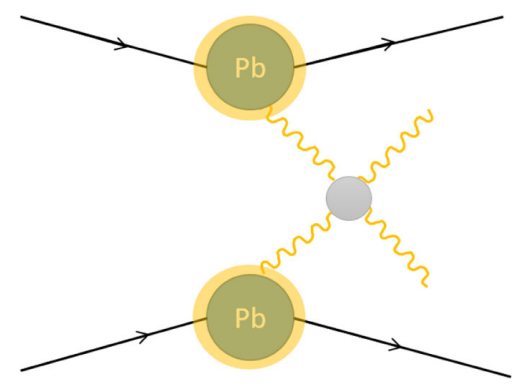

FIG. 1. Sketch of light-by-light scattering through photonphoton collisions in ultraperipheral $\mathrm{Pb}-\mathrm{Pb}$ collisions.

charged particle to be treated as equivalent to a flux of on-shell photons. Since the photon flux is proportional to $Z^{2}$ for each nucleus, the coherent enhancement in the exclusive $\gamma \gamma$ cross section scales as $Z^{4}$, where $Z=82$ for the lead $(\mathrm{Pb})$ ions used at the LHC. This is why heavy-ion collisions have an advantage over proton-proton or protonlead collisions for probing physics in electromagnetic processes [8]. Photon fusion in ultraperipheral heavy-ion collisions has been suggested as a way of detecting the Higgs boson [28,29], and, more recently, the possibility of constraining new physics beyond the standard model (BSM) in this process was studied in Refs. [30,31].

As was already mentioned, the possibility of directly observing light-by-light scattering at the LHC was proposed in Ref. [8], and this long-standing prediction of QED was finally measured earlier this year with $4.4 \sigma$ significance by the ATLAS Collaboration [9] at a level in good agreement with calculations in Refs. $[8,10]$. The compatibility with the standard model constrains any possible contributions from BSM physics. Born-Infeld theory is particularly interesting in this regard, as constraints from low-energy optical and atomic experiments have yet to reach the sensitivity of interest for measuring light-by-light scattering $[19,20]$.

The leading-order cross section for unpolarized light-bylight scattering in Born-Infeld theory in the $\gamma \gamma$ center-ofmass frame is given by $[17,32]$

$$
\sigma_{\mathrm{BI}}(\gamma \gamma \rightarrow \gamma \gamma)=\frac{1}{2} \int d \Omega \frac{d \sigma_{\mathrm{BI}}}{d \Omega}=\frac{7}{1280 \pi} \frac{m_{\gamma \gamma}^{6}}{\beta^{4}},
$$

where $m_{\gamma \gamma}$ is the diphoton invariant mass and the differential cross section is

$$
\frac{d \sigma_{\mathrm{BI}}}{d \Omega}=\frac{1}{4096 \pi^{2}} \frac{m_{\gamma \gamma}^{6}}{\beta^{4}}(3+\cos \theta)^{2} .
$$

We recall that the parameter $\beta=M^{2}$ enters as a dimensionful parameter in the Born-Infeld theory of nonlinear QED defined by the Lagrangian (1). If this originates from a Born-Infeld theory of hypercharge, then the corresponding mass scale is $M_{Y}=\cos \theta_{W} M$.

We plot in Fig. 2 the angular distributions as functions of $\cos \theta$ in the center-of-mass frame (where $\theta$ is the polar angle) for the leading-order differential cross sections in both Born-Infeld theory and QED (with arbitrary 


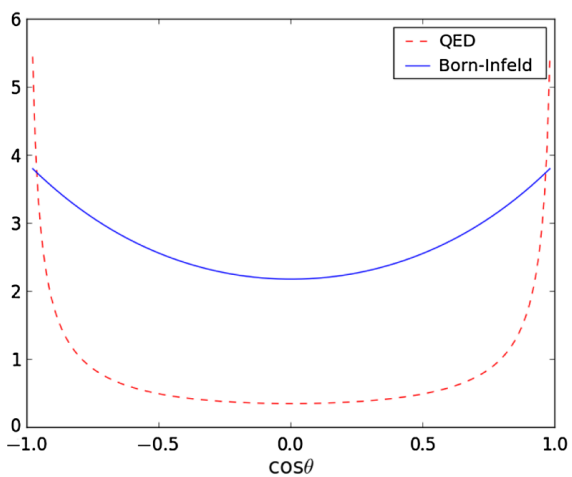

FIG. 2. Comparison between the angular distributions (with arbitrary normalizations) as functions of $\cos \theta$ in the center-ofmass frame (where $\theta$ is the polar angle) for the leading-order differential cross sections in $\mathrm{U}(1)_{\mathrm{EM}}$ Born-Infeld theory and QED, plotted as solid blue and dashed red lines, respectively.

normalizations), as solid blue and dashed red lines, respectively. We see that the Born-Infeld distribution is less forward peaked than that for QED. For the latter, we used the leading-order amplitudes for the quark and lepton box loops in the ultrarelativistic limit from Ref. [33], omitting the percent-level effects of higher-order QCD and QED corrections, as well the $W^{ \pm}$contribution that is negligible for typical diphoton center-of-mass masses at the LHC.

The total exclusive diphoton cross section from $\mathrm{Pb}+\mathrm{Pb}$ collisions is obtained by convoluting the $\gamma \gamma \rightarrow \gamma \gamma$ cross section with a luminosity function $d L / d \tau$ [34],

$$
\sigma_{\mathrm{excl}}=\int_{\tau_{0}}^{1} d \tau \frac{d L}{d \tau} \sigma_{\gamma \gamma \rightarrow \gamma \gamma}(\tau)
$$

We have introduced here a dimensionless measure of the diphoton invariant mass, $\tau \equiv m_{\gamma \gamma}^{2} / s_{N N}$, where $\sqrt{s_{N N}}=$ $5.02 \mathrm{TeV}$ is the center-of-mass energy per nucleon pair in the ATLAS measurement. The luminosity function, derived, for example, in Ref. [34], can be written as an integral over the number distribution of photons carrying a fraction $x$ of the total $\mathrm{Pb}$ momentum:

$$
\frac{d L}{d \tau}=\int_{\tau}^{1} d x_{1} d x_{2} f\left(x_{1}\right) f\left(x_{2}\right) \delta\left(\tau-x_{1} x_{2}\right),
$$

where the distribution function $f(x)$ depends on a nuclear form factor. We follow Ref. [34] in adopting the form factor proposed in Ref. [29], while noting that variations in the choice leads to $\sim 20 \%$ uncertainties in the final cross sections [8]. A contribution with a nonfactorizable distribution function should also be subtracted to account for the exclusion of nuclear overlaps, but this is not a significant effect for the relevant kinematic range, causing a difference within the $20 \%$ uncertainty [31] from the photon luminosity evaluated numerically using the STARLIGHT code [35]. For $\sqrt{s_{N N}}=5.5 \mathrm{TeV}$ and $m_{\gamma \gamma}>5 \mathrm{GeV}$, we obtain a QED cross section of $\sigma_{\text {excl }}^{\mathrm{QED}}=385 \pm 77 \mathrm{nb}$, in good agreement with Ref. [8]. The ATLAS measurement is performed at $\sqrt{s_{N N}}=5.02 \mathrm{TeV}$ and for $m_{\gamma \gamma}>6 \mathrm{GeV}$, for which we find $\sigma_{\mathrm{excl}}^{\mathrm{QED}}=220 \pm 44 \mathrm{nb}$.

This total $\gamma \gamma \rightarrow \gamma \gamma$ cross section is reduced by the fiducial cuts of the ATLAS analysis, which restrict the phase space to a photon pseudorapidity region $|\eta|<2.4$ and require photon transverse energies $E_{T}>3 \mathrm{GeV}$ and the diphoton system to have an invariant mass $m_{\gamma \gamma}>6 \mathrm{GeV}$, with a transverse momentum $p_{T}^{\gamma \gamma}<$ $2 \mathrm{GeV}$ and an acoplanarity Aco $=1-\Delta \phi / \pi<0.01$. We simulate the event selection using Monte Carlo sampling, implementing the cuts with a 15\% Gaussian smearing in the photon transverse energy resolution at low energies and $0.7 \%$ at higher energies $[9,36]$ above $100 \mathrm{GeV}$. Since the differential cross section does not depend on $\phi$, we implement the acoplanarity cut as a fixed $85 \%$ efficiency in the number of signal events after the $p_{T}^{\gamma \gamma}$ selection, following the ATLAS analysis [9]. The total reduction in yield for the QED case is a factor $\epsilon \sim 0.30$, which results in a fiducial cross section $\sigma_{\text {fid }}^{\text {QED }}=53 \pm 11 \mathrm{nb}$ for $\sqrt{s}_{N N}=5.02 \mathrm{TeV}$, in good agreement with the two predictions of 45 and $49 \mathrm{nb}$ quoted by ATLAS [9].

Following this validation for the QED case, we repeat the procedure for the Born-Infeld cross section. Since the BornInfeld $\gamma \gamma \rightarrow \gamma \gamma$ cross section grows with energy, the dominant contribution to the cross section comes from the $\tau \lesssim 0.2$ part of the integral, compared with $\tau \lesssim 10^{-4}$ for the QED case. We show in Fig. 3 the distributions of the $\sigma(\gamma \gamma \rightarrow \gamma \gamma)$ cross section multiplied by the photon flux luminosity factor-normalized by the total exclusive cross sectionas functions of the invariant diphoton mass distribution, for the QED case in the upper panel and in Born-Infeld theory with $M=\sqrt{\beta}=200 \mathrm{GeV}$ in the lower panel.

We see that the invariant-mass distribution in the BornInfeld case extends to $m_{\gamma \gamma}>M$, where the validity of the tree-level Born-Infeld Lagrangian may be questioned because the Taylor expansion of the square root in the nonpolynomial Born-Infeld Lagrangian (1) could break down. With this in mind, we use two approaches to place plausible limits on $M=\sqrt{\beta}$. In the first and most conservative method, we consider $\gamma \gamma$ scattering only for $m_{\gamma \gamma} \leq M$, while in the second approach we integrate the $\gamma \gamma$ cross section (4) up to the diphoton invariant mass where the unitarity limit $\sigma_{\mathrm{BI}} \sim 1 / m_{\gamma \gamma}^{2}$ is saturated, beyond which we assume that the cross section saturates the unitarity limit and falls as $\sim 1 / m_{\gamma \gamma}^{2}$.

We find fiducial efficiencies for the cutoff and unitarization approaches to be $\epsilon \sim 0.39$ and 0.14 , respectively. While the $E_{T}$ and $\eta$ cuts have much less effect than for QED, as expected from the difference in the angular distributions visible in Fig. 2, the larger invariant masses appearing in the Born-Infeld case are much more affected by the $p_{T}^{\gamma \gamma}$ requirement.

Our calculations of the corresponding $\mathrm{U}(1)_{\mathrm{EM}}$ BornInfeld fiducial cross sections are plotted in the upper 

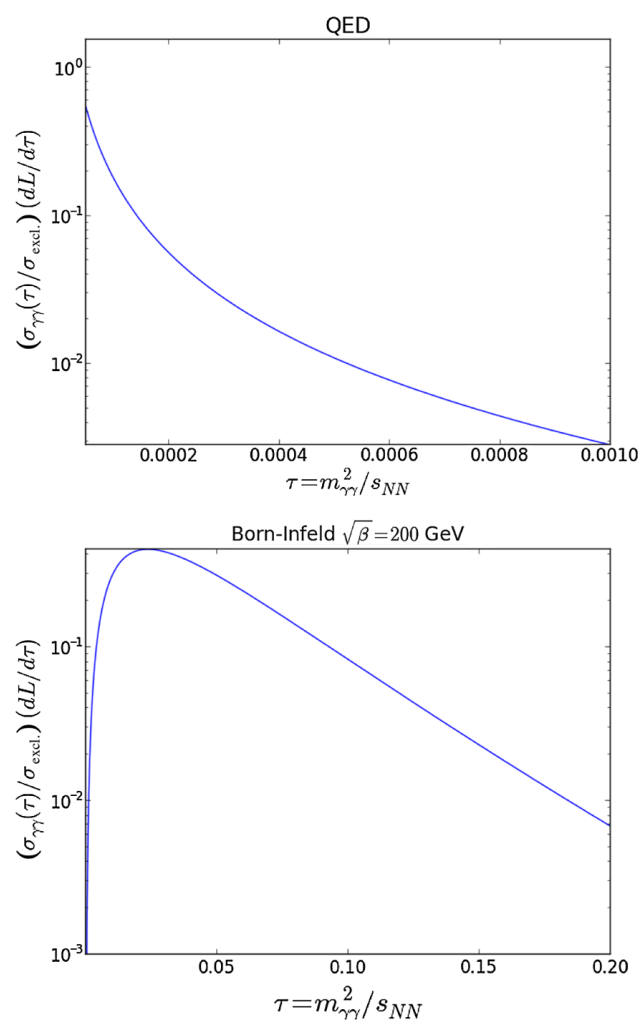

FIG. 3. The distributions in the scaled diphoton invariant mass $\tau \equiv m_{\gamma \gamma}^{2} / s_{N N}$, normalized by the total $\gamma \gamma \rightarrow \gamma \gamma$ cross section, for the QED case in the upper panel and for $\mathrm{U}(1)_{\mathrm{EM}}$ Born-Infeld theory with $M=\sqrt{\beta}=200 \mathrm{GeV}$ in the lower panel.

panel of Fig. 4 as a function of $M=\sqrt{\beta}$ : the green curve is for the more conservative cutoff approach, and the blue curve assumes that unitarity is saturated. These calculations are confronted with the ATLAS measurement of $\sigma_{\text {fid }}=70 \pm 24$ (stat) \pm 17 (syst) nb [9], assuming that these errors are Gaussian and adding them in quadrature with a theory uncertainty of $\pm 10 \mathrm{nb}$. We perform a $\chi^{2}$ fit to obtain the $95 \%$ C.L. upper limit on a Born-Infeld signal additional to the $49 \mathrm{nb}$ standard model prediction. (We neglect possible interference effects that are expected to be small due to the different invariant-mass and angular distributions involved.) This corresponds to the excluded range shaded in pink above $\sigma_{\text {fid }}^{95 \% \text { C.L. }} \sim 65 \mathrm{nb}$ in the upper panel of Fig. 4, which translates to the limit $M=\sqrt{\beta} \gtrsim$ $100(190) \mathrm{GeV}$ in the cutoff (unitarized) approach, as indicated by the green (blue) vertical dashed line in Fig. 4.

These limits could be strengthened further by considering the $m_{\gamma \gamma}$ distribution shown in Fig. 3(b) of Ref. [9], where we see that all of the observed events had $m_{\gamma \gamma}<25 \mathrm{GeV}$, in line with expectations in QED, whereas, in the Born-Infeld theory, most events would have $m_{\gamma \gamma}>25 \mathrm{GeV}$. Calculating a ratio of the total exclusive cross section of QED for $m_{\gamma \gamma}>6 \mathrm{GeV}$ and $>25 \mathrm{GeV}$ as $\sigma_{\text {excl }}^{m_{\gamma y}>25 \mathrm{GeV}} / \sigma_{\text {excl }}^{m_{\gamma \gamma}>6 \mathrm{GeV}} \sim 0.02$, we estimate a $95 \%$ C.L.
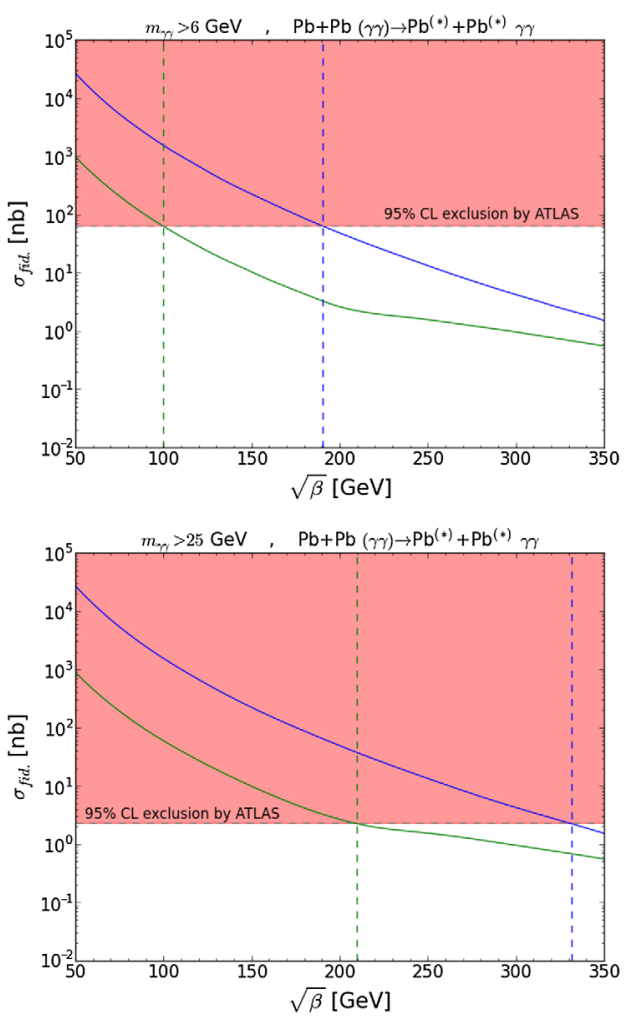

FIG. 4. The fiducial cross section for light-by-light scattering in relativistic heavy-ion collisions, $\sigma\left(\mathrm{Pb}+\mathrm{Pb}(\gamma \gamma) \rightarrow \mathrm{Pb}^{(*)}+\right.$ $\left.\mathrm{Pb}^{(*)} \gamma \gamma\right)$ as a function of $M=\sqrt{\beta}$ in the $\mathrm{U}(1)_{\mathrm{EM}}$ Born-Infeld theory is shown as a solid green (blue) line for a hard cutoff (unitarized) approach, as discussed in the text. The lower diphoton invariant mass cutoff is set at $6 \mathrm{GeV}(25 \mathrm{GeV})$ on the upper (lower) plot. This is compared with the 95\% C.L. upper limit obtained from the ATLAS measurement [9] by combining the statistical and systematic errors in quadrature, as well as a $10 \mathrm{nb}$ theoretical uncertainty in the cross section predicted in QED $[8,10]$ (the horizontal dashed line), which excludes the higher range shaded pink. The corresponding 95\% C.L. lower limits $M \gtrsim 100(190) \mathrm{GeV}$ for $m_{\gamma \gamma}>6 \mathrm{GeV}$ and $M \gtrsim$ $210(330) \mathrm{GeV}$ for $m_{\gamma \gamma}>25 \mathrm{GeV}$ are shown as vertical dashed lines in green (blue).

upper limit of $\sim 2 \mathrm{nb}$ for $m_{\gamma \gamma}>25 \mathrm{GeV}$. The corresponding exclusion plot is shown in the lower panel of Fig. 4, where we see a stronger limit $M=\sqrt{\beta} \gtrsim 210(330) \mathrm{GeV}$ in the cutoff (unitarized) approach, with the same color coding used previously.

Our lower limit on the QED Born-Infeld scale $M=$ $\sqrt{\beta} \gtrsim 100 \mathrm{GeV}$ is at least 3 orders of magnitude stronger than the lower limits on $M=\sqrt{\beta}$ obtained from previous measurements of nonlinearities in light [14-17,19,20]. Because of the kinematic cuts made in the ATLAS analysis, our limit does not apply to a range of values of $M \lesssim$ $10 \mathrm{GeV}$ for which the nonlinearities in Eq. (1) should be taken into account. However, our limit is the first to approach the range of potential interest for string or $M$ theory constructions since models with (stacks of) branes 
separated by distances $1 / M: M=O(1) \mathrm{TeV}$ have been proposed in that context [37]. Our analysis could clearly be refined with more sophisticated detector simulations and the uncertainties reduced. However, in view of the strong power-law dependence of the Born-Infeld cross section on $M=\sqrt{\beta}$ visible in Eq. (4), the scope for significant improvement in our constraint is limited unless experiments can probe substantially larger $m_{\gamma \gamma}$ ranges. In this regard, it would be interesting to explore the sensitivities of high-energy $e^{+} e^{-}$machines considered as $\gamma \gamma$ colliders.

As mentioned in the introduction, Arunasalam and Kobakhidze recently pointed out [21] that the standard model modified by a Born-Infeld theory of the hypercharge $\mathrm{U}(1)_{Y}$ contains a finite-energy monopole solution with mass $M_{\mathcal{M}}=E_{0}+E_{1}$, where $E_{0}$ is the contribution associated with the Born-Infeld $\mathrm{U}(1)_{Y}$ hypercharge, and $E_{1}$ is associated with the remainder of the Lagrangian. Arunasalam and Kobakhidze estimated [21] that $E_{0} \simeq 72.8 M_{Y}$, where $M_{Y}=\cos \theta_{W} M$, and Cho et al. had previously estimated [23] that $E_{1} \simeq 4 \mathrm{TeV}$. (Both of these estimates are at the classical level, and quantum corrections have yet to be explored.) Combining these calculations and using our lower limit $M \gtrsim 100 \mathrm{GeV}$ (2), we obtain a lower limit $M_{\mathcal{M}} \gtrsim$ $11 \mathrm{TeV}$ on the $\mathrm{U}(1)_{Y}$ Born-Infeld monopole mass. (For completeness, we recall that it was argued in Ref. [21] that nucleosynthesis constraints on the abundance of relic monopoles require $M_{\mathcal{M}} \lesssim 23 \mathrm{TeV}$.) Unfortunately, this is beyond the reach of MoEDAL [26] or any other experiment at the LHC [25], but it could lie within reach of a similar experiment at any future $100-\mathrm{TeV} p p$ collider [38], or of a cosmic ray experiment.

In this Letter we have restricted our attention to possible Born-Infeld modifications of $U(1)$ gauge factors and their constraints in light-by-light scattering only. We plan to examine in the future the experimental constraints from measurements at the LHC on possible Born-Infeld extensions of the $\mathrm{SU}(3)_{C}$ and $\mathrm{SU}(2)_{L}$ gauge symmetries of the standard model.

The work of J. E. and N.E. M. was supported partly by STFC Grant No. ST/L000326/1. The work of T. Y. was supported by a Junior Research Fellowship from Gonville and Caius College, Cambridge, England. We thank Vasiliki Mitsou for drawing our attention to Ref. [21], and her and Albert De Roeck, Igor Ostrovskiy, and Jim Pinfold of the MoEDAL Collaboration for their interest and the relevant discussions. T. Y. is grateful for the hospitality of King's College London, where part of this work was completed.

[1] P. A. M. Dirac, The quantum theory of the electron, Proc. R. Soc. A 117, 610 (1928).

[2] P. A. M. Dirac, Quantized singularities in the electromagnetic field, Proc. R. Soc. A 133, 60 (1931).

[3] O. Halpern, Scattering processes produced by electrons in negative energy states, Phys. Rev. 44, 855 (1933).
[4] W. Heisenberg, Bemerkungen zur Diracschen Theorie des Positrons, Z. Phys. 90, 209 (1934); Erratum, Z. Phys. 92, 692(E) (1934).

[5] H. Euler and B. Kockel, Über die Streuung von Licht an Licht nach der Diracschen Theorie, Naturwissenschaften 23, 246 (1935).

[6] W. Heisenberg and H. Euler, Consequences of Dirac's theory of positrons, Z. Phys. 98, 714 (1936).

[7] R. Karplus and M. Neuman, The scattering of light by light, Phys. Rev. 83, 776 (1951).

[8] D. d'Enterria and G. G. da Silveira, Observing Light-byLight Scattering at the Large Hadron Collider, Phys. Rev. Lett. 111, 080405 (2013); Erratum, Phys. Rev. Lett. 116, 129901(E) (2016)].

[9] M. Aaboud et al. (ATLAS Collaboration), Evidence for light-by-light scattering in heavy-ion collisions with the ATLAS detector at the LHC, arXiv:1702.01625.

[10] M. Kłusek-Gawenda, P. Lebiedowicz, and A. Szczurek, Light-by-light scattering in ultraperipheral $\mathrm{Pb}-\mathrm{Pb}$ collisions at energies available at the CERN Large Hadron Collider, Phys. Rev. C 93, 044907 (2016).

[11] M. Born and L. Infeld, Foundations of the new field theory, Proc. R. Soc. A 144, 425 (1934).

[12] E. S. Fradkin and A. A. Tseytlin, Nonlinear electrodynamics from quantized strings, Phys. Lett. 163B, 123 (1985).

[13] C. Bachas, D-brane dynamics, Phys. Lett. B 374, 37 (1996).

[14] J. Rafelski, G. Soff, and W. Greiner, Lower bound to limiting fields in nonlinear electrodynamics, Phys. Rev. A 7, 903 (1973).

[15] H. Carley and M. K.-H. Kiessling, Nonperturbative Calculation of Born-Infeld Effects on the Schrödinger Spectrum of the Hydrogen Atom, Phys. Rev. Lett. 96, 030402 (2006).

[16] S.Z. Akhmadaliev et al., Experimental Investigation of High-Energy Photon Splitting in Atomic Fields, Phys. Rev. Lett. 89, 061802 (2002).

[17] J. M. Dávila, C. Schubert, and M. A. Trejo, Photonic processes in Born-Infeld theory, Int. J. Mod. Phys. A 29, 1450174 (2014).

[18] S. Mereghetti, The strongest cosmic magnets: Soft gammaray repeaters and anomalous $\mathrm{x}$-ray pulsars, Astron. Astrophys. Rev. 15, 225 (2008).

[19] F. Della Valle, E. Milotti, A. Ejlli, G. Messineo, L. Piemontese, G. Zavattini, U. Gastaldi, R. Pengo, and G. Ruoso, First results from the new PVLAS apparatus: A new limit on vacuum magnetic birefringence, Phys. Rev. D 90, 092003 (2014).

[20] M. Fouché, R. Battesti, and C. Rizzo, Limits on nonlinear electrodynamics, Phys. Rev. D 93, 093020 (2016).

[21] S. Arunasalam and A. Kobakhidze, Electroweak monopoles and the electroweak phase transition, arXiv:1702.04068.

[22] Y. M. Cho and D. Maison, Monopoles in Weinberg-Salam model, Phys. Lett. B 391, 360 (1997).

[23] Y. M. Cho, K. Kim, and J. H. Yoon, Finite energy electroweak dyon, Eur. Phys. J. C 75, 240 (2015).

[24] J. Ellis, N. E. Mavromatos, and T. You, The price of an electroweak monopole, Phys. Lett. B 756, 29 (2016).

[25] G. Aad et al. (ATLAS Collaboration), Search for Magnetic Monopoles in $\sqrt{s}=7 \mathrm{TeV} p p$ Collisions with the ATLAS Detector, Phys. Rev. Lett. 109, 261803 (2012); Search for 
magnetic monopoles and stable particles with high electric charges in $8 \mathrm{TeV} p p$ collisions with the ATLAS detector, Phys. Rev. D 93, 052009 (2016).

[26] B. Acharya et al. (MoEDAL Collaboration), The physics programme of the MoEDAL experiment at the LHC, Int. J. Mod. Phys. A 29, 1430050 (2014); Search for magnetic monopoles with the MoEDAL prototype trapping detector in $8 \mathrm{TeV}$ proton-proton collisions at the LHC, J. High Energy Phys. 08 (2016) 067; Search for Magnetic Monopoles with the MoEDAL Forward Trapping Detector in $13 \mathrm{TeV}$ Proton-Proton Collisions at the LHC, Phys. Rev. Lett. 118, 061801 (2017).

[27] C. von Weizsacker, Ausstrahlung bei Stößen sehr schneller Elektronen, Z. Phys. 88, 612 (1934); E. J. Williams, Nature of the high energy particles of penetrating radiation and status of ionization and radiation formulae, Phys. Rev. 45, 729 (1934); E. Fermi, Sulla teoria dell' urto tra atomi e corpuscoli elettrici, Nuovo Cimento 2, 143 (1925).

[28] E. Papageorgiu, Coherent Higgs-boson production in relativistic heavy-ion collisions, Phys. Rev. D 40, 92 (1989).

[29] M. Drees, J. R. Ellis, and D. Zeppenfeld, Can one detect an intermediate mass Higgs boson in heavy ion collisions?, Phys. Lett. B 223, 454 (1989).

[30] S. Fichet, Shining light on polarizable dark particles, J. High Energy Phys. 04 (2017) 088.
[31] S. Knapen, T. Lin, H. K. Lou, and T. Melia, Searching for Axionlike Particles with Ultraperipheral Heavy-Ion Collisions, Phys. Rev. Lett. 118, 171801 (2017).

[32] A. Rebhan and G. Turk, Polarization effects in light-by-light scattering: Euler-Heisenberg versus Born-Infeld, Int. J. Mod. Phys. A 32, 1750053 (2017).

[33] Z. Bern, A. De Freitas, L. J. Dixon, A. Ghinculov, and H. L. Wong, QCD and QED corrections to light by light scattering, J. High Energy Phys. 11 (2001) 031.

[34] R. N. Cahn and J. D. Jackson, Realistic equivalent photon yields in heavy ion collisions, Phys. Rev. D 42, 3690 (1990).

[35] S. R. Klein, J. Nystrand, J. Seger, Y. Gorbunov, and J. Butterworth, STARLIGHT: A Monte Carlo simulation program for ultra-peripheral collisions of relativistic ions, Comput. Phys. Commun. 212, 258 (2017); see https:// starlight.hepforge.org.

[36] G. Aad et al. (ATLAS Collaboration), Electron and photon energy calibration with the ATLAS detector using LHC Run 1 data, Eur. Phys. J. C 74, 3071 (2014).

[37] For a review and references, see A. A. Tseytlin, in The Many Faces of the Superworld, edited by M. A. Shifman (World Scientific, Singapore, 2000), p. 417.

[38] See, for example, the Future Circular Collider Study, https:// fcc.web.cern.ch/Pages/default.aspx. 